\title{
Subsystem Interpolation for Parameterized Bilinear Dynamical Systems
}

\author{
A. Carracedo Rodriguez, Serkan Gugercin, Jeff Borggaard \\ Mathematics Department, Virginia Tech, Blacksburg, VA 24061 USA
}

(e-mail: $\{$ crandrea,gugercin,jborggaard\}@vt.edu)

\section{INTRODUCTION}

Consider the following parametric bilinear system

$$
\left\{\begin{array}{l}
\dot{\mathbf{x}}(t)=\mathbf{A}(p) \mathbf{x}(t)+\mathbf{N}(p) \mathbf{x}(t) u(t)+\mathbf{b}(p) u(t), \\
y(t)=\mathbf{c}^{\top}(p) \mathbf{x}(t)
\end{array}\right.
$$

where $\mathbf{x}(t) \in \mathbb{R}^{n}, y(t) \in \mathbb{R}$, and $u(t) \in \mathbb{R}$ denote the states, output, and input, respectively. We assume that the parameter $p$ is a scalar, i.e., $p \in \mathbb{R}$. We want to find a lower dimensional parametric bilinear system whose output is a good approximation to the output of the full order system for a wide variety of inputs $u(t)$ and parameter values $p$. We do so by means of a Petrov-Galerkin projection, i.e., given the reduction bases $\mathbf{V} \in \mathbb{R}^{n \times r}$ and $\mathbf{W} \in \mathbb{R}^{n \times r}$ with $\mathbf{W}^{\top} \mathbf{V}=\mathbf{I}_{r}$ where $r \ll n$. The reduced model has the same structure as in (1) with reduced matrices given by:

$$
\begin{aligned}
\widetilde{\mathbf{A}}(p)=\mathbf{W}^{\top} \mathbf{A}(p) \mathbf{V} \in \mathbb{R}^{r \times r}, & \widetilde{\mathbf{b}}(p)=\mathbf{W}^{\top} \mathbf{b}(p) \in \mathbb{R}^{r \times 1} \\
\widetilde{\mathbf{N}}(p)=\mathbf{W}^{\top} \mathbf{N}(p) \mathbf{V} \in \mathbb{R}^{r \times r}, & \widetilde{\mathbf{c}}(p)=\mathbf{V}^{\top} \mathbf{c}(p) \in \mathbb{R}^{r \times 1} .
\end{aligned}
$$

For the recomputations of the reduced model to be efficient, we assume an affine structure on the matrices in (1) with respect to the parameter. For example, $\mathbf{A}(p)$ is assumed to have the form

$$
\mathbf{A}(p)=\mathbf{A}_{0}+\sum_{i=1}^{\ell} f_{i}(p) \mathbf{A}_{i}
$$

where $\mathbf{A}_{i} \in \mathbb{R}^{n \times n}$ are constant matrices and $f_{i}(p)$ are scalar nonlinearities, for $i=1, \ldots, \ell$. We will focus on building $\mathbf{V}$ and $\mathbf{W}$ so that interpolation of the two first transfer functions of (1) is guaranteed. Thus the goal is to extend the interpolatory parametric model reduction results of Baur et al. [2011] to the subsystem bilinear interpolation framework of Breiten\&Damm [2010]. For more details on parametric and nonparametric model redution see Benner et al. [2015], Antoulas [2005], Antoulas et al. [2001], Baur et al. [2014], Benner et al. [2017], Benner et al. [2017], Hesthaven et al. [2016], Quarteroni et al. [2016], Benner and Breiten [2012], Flagg and Gugercin [2015], and references therein.

The two leading subsystem transfer functions of the bilinear system (1) are given by

\footnotetext{
* Supported in part by the National Science Foundation under contract DMS1522616 and the National Institute for Occupational Safety and Health under contract 200-2014-59669. The work of Gugercin was also supported in part by the Alexander von Humboldt Foundation.
}

$$
\begin{aligned}
& \mathbf{H}_{1}(s ; p)=\mathbf{c}^{\top}(p) \mathbf{K}(s ; p) \mathbf{b}(p), \quad \text { and } \\
& \mathbf{H}_{2}\left(s_{1}, s_{2} ; p\right)=\mathbf{c}^{\top}(p) \mathbf{K}\left(s_{2} ; p\right) \mathbf{N}(p) \mathbf{K}\left(s_{1} ; p\right) \mathbf{b}(p)
\end{aligned}
$$

where

$$
\mathbf{K}(s ; p)=\left(s \mathbf{I}_{n}-\mathbf{A}(p)\right)^{-1} .
$$

The subsystem transfer functions of the reduced bilinear system are defined similarly and denoted by $\widetilde{\mathbf{H}}_{1}$ and $\widetilde{\mathbf{H}}_{2}$.

\section{MAIN RESULTS}

We now list the main results that show how to construct $\mathbf{V}$ and $\mathbf{W}$ for desired interpolation conditions:

Theorem 1. Let $\left\{\sigma_{1}, \sigma_{2}\right\} \subset \mathbb{C}$ and $\hat{p} \in \mathbb{R}$ such that $\mathbf{K}\left(\sigma_{i} ; \hat{p}\right)$ exists for all $i \in\{1,2\}$. Define

$$
\begin{aligned}
& \mathbf{v}_{1}=\mathbf{K}\left(\sigma_{1} ; \hat{p}\right) \mathbf{b}(\hat{p}), \quad \mathbf{v}_{2}=\mathbf{K}\left(\sigma_{2} ; \hat{p}\right) \mathbf{N}(\hat{p}) \mathbf{v}_{1}, \\
& \mathbf{w}_{1}=\mathbf{K}\left(\sigma_{2} ; \hat{p}\right)^{\top} \mathbf{c}(\hat{p}), \quad \mathbf{w}_{2}=\mathbf{K}\left(\sigma_{1} ; \hat{p}\right)^{\top} \mathbf{N}(\hat{p})^{\top} \mathbf{w}_{1} . \\
&\left\{\mathbf{v}_{1}, \mathbf{v}_{2}\right\} \subseteq \mathbf{R a n} \mathbf{V},
\end{aligned}
$$
If

then

If

$$
\begin{aligned}
& \mathbf{H}_{1}\left(\sigma_{1} ; \hat{p}\right)=\widetilde{\mathbf{H}}_{1}\left(\sigma_{1} ; \hat{p}\right), \\
& \mathbf{H}_{2}\left(\sigma_{1}, \sigma_{2} ; \hat{p}\right)=\widetilde{\mathbf{H}}_{2}\left(\sigma_{1}, \sigma_{2} ; \hat{p}\right) .
\end{aligned}
$$

then

$$
\left\{\mathbf{w}_{1}, \mathbf{w}_{2}\right\} \subseteq \operatorname{Ran} \mathbf{W}
$$

$$
\begin{aligned}
& \mathbf{H}_{1}\left(\sigma_{2} ; \hat{p}\right)=\widetilde{\mathbf{H}}_{1}\left(\sigma_{2} ; \hat{p}\right), \\
& \mathbf{H}_{2}\left(\sigma_{1}, \sigma_{2} ; \hat{p}\right)=\widetilde{\mathbf{H}}_{2}\left(\sigma_{1}, \sigma_{2} ; \hat{p}\right) .
\end{aligned}
$$

Theorem 2. Assume the conditions in Theorem 1. If both (7) and (9) hold, then not only do we have interpolation of the transfer functions

$$
\begin{aligned}
& \mathbf{H}_{1}\left(\sigma_{1} ; \hat{p}\right)=\widetilde{\mathbf{H}}_{1}\left(\sigma_{1} ; \hat{p}\right), \\
& \mathbf{H}_{2}\left(\sigma_{1}, \sigma_{2} ; \hat{p}\right)=\widetilde{\mathbf{H}}_{2}\left(\sigma_{1}, \sigma_{2} ; \hat{p}\right),
\end{aligned}
$$

but also of their sensitivities, i.e.

$$
\begin{aligned}
\frac{\partial}{\partial s} \mathbf{H}_{1}\left(\sigma_{1} ; \hat{p}\right) & =\frac{\partial}{\partial s} \widetilde{\mathbf{H}}_{1}\left(\sigma_{1} ; \hat{p}\right), \\
\frac{\partial}{\partial s_{i}} \mathbf{H}_{2}\left(\sigma_{1}, \sigma_{2} ; \hat{p}\right) & =\frac{\partial}{\partial s_{i}} \widetilde{\mathbf{H}}_{2}\left(\sigma_{1}, \sigma_{2} ; \hat{p}\right), \\
\frac{\partial}{\partial p} \mathbf{H}_{1}\left(\sigma_{1} ; \hat{p}\right) & =\frac{\partial}{\partial p} \widetilde{\mathbf{H}}_{1}\left(\sigma_{1} ; \hat{p}\right), \\
\frac{\partial}{\partial p} \mathbf{H}_{2}\left(\sigma_{1}, \sigma_{2} ; \hat{p}\right) & =\frac{\partial}{\partial p} \widetilde{\mathbf{H}}_{2}\left(\sigma_{1}, \sigma_{2} ; \hat{p}\right) .
\end{aligned}
$$

Remark. Interpolation of higher order derivatives can be attained by including derivative information in the 
reduction bases. We skip these details together with the proofs of above theorem for conciseness. The complete case of multi-input/multi-output systems together with a parameter vector $\mathbf{p}$ (as opposed to scalar $p$ considered here) can be found in Carracedo et al. [2017].

\section{EXAMPLE}

Consider the following model of the transport and diffusion of the temperature of a fluid with thermal conductivity $\kappa$ on the domain $\Omega=[-1,1] \times[-1,1]$ :

$$
\begin{array}{ll}
\dot{T}=\kappa \Delta T-\mathbf{v} \cdot \nabla T+u(t) f \\
T=0 & \text { at } t=0 \\
T=1 & \text { on } \partial \Omega
\end{array}
$$

where

$$
\mathbf{v}(x, y)=\sin t\left[\begin{array}{c}
-y \\
x
\end{array}\right]+\frac{\cos t}{2}(\cos (\pi(x-y))+1)\left[\begin{array}{l}
1 \\
1
\end{array}\right]
$$

and

$$
f(x, y)=\exp \left(-\left(x^{2}+y^{2}\right)\right)
$$

is a source with strength controlled by the input $u(t)$. We can rewrite this model as a parametric bilinear system with input $u(t)$ and output (of our choice) the average temperature over $[0.5,1] \times[0.5,1]$. Then we can define a reduced-order model as in (2) with basis satisfying the conditions in Theorem 2. We do so for two different parameter sets: in the first set we only sample at 0.05 ; and in the second set we sample at $0.05,0.06,0.07$, and 0.08. We show the performance of the reduced model for a non-sampled parameter value in Figures 1 and 2. Note that the full-order model has dimension 361 while the reduced-order model in Figures 1 and 2 has dimension 6 and 12, respectively. The figures show that a good approximation may be attained with this method by sampling the parameter space appropriately.

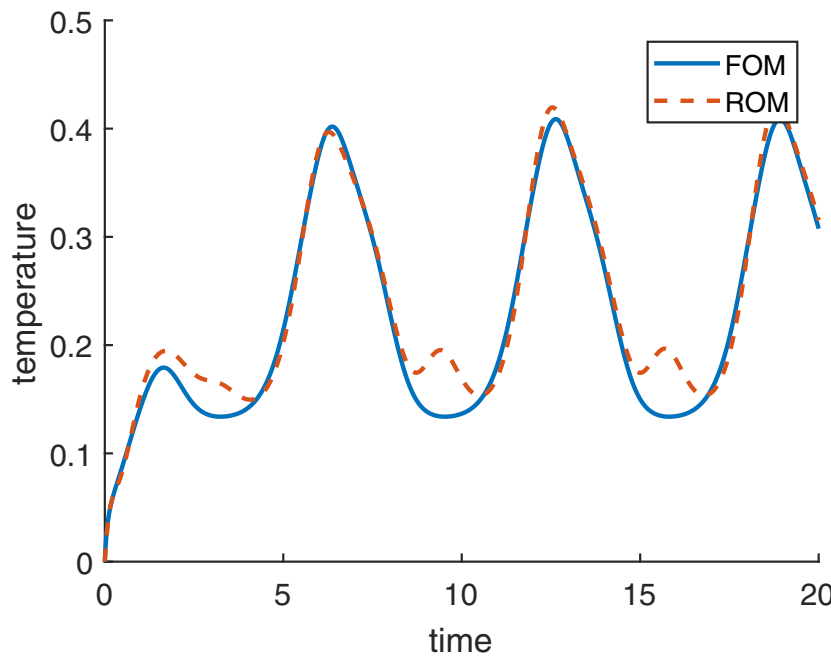

Fig. 1. Solution of the full order model and the reduced order model for parameter value $\kappa=0.055$ and input $u(t)=0.5$. Parameter sample: $\hat{\kappa}=0.05$.

\section{REFERENCES}

Antoulas. Approximation of large-scale dynamical systems. SIAM Publications, Philadelphia, PA, 2005.

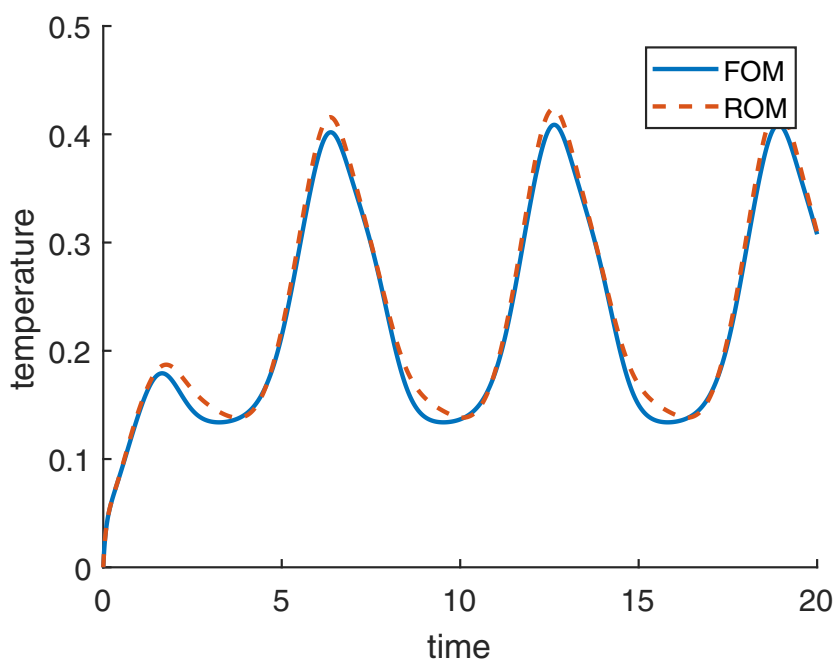

Fig. 2. Solution of the full order model and the reduced order model for parameter value $\kappa=0.055$ and input $u(t)=0.5$. Parameter samples: $\hat{\kappa}_{1}=0.05, \hat{\kappa}_{2}=0.06$, $\hat{\kappa}_{3}=0.07, \hat{\kappa}_{4}=0.08$.

Antoulas, Sorensen, and Gugercin. A survey of model reduction methods for large-scale systems. Contemporary Mathematics 280:193-219, 2001.

Baur, Beattie, Benner, and Gugercin. Interpolatory projection methods for parameterized model reduction. SIAM Journal on Scientific Computing, 33(5):24892518, 2011.

Baur, Benner, and Feng. Model order reduction for linear and nonlinear systems: A system-theoretic perspective. Arch. Comput. Methods Eng. 21(4):331-358, 2014.

Benner and Breiten. Interpolation-based $\mathcal{H}_{2}$-model reduction of bilinear control systems. SIAM Journal on Matrix Analysis \& Applications, 33(3):859-885, 2012.

Benner, Gugercin, and Willcox. A survey of projectionbased model reduction for parametric dynamical systems. SIAM Review, 57(4):483-531, 2015.

Benner, Cohen, Ohlberger, Willcox. Model reduction and approximation: theory and algorithms. SIAM, 2017.

Benner, Ohlberger, Patera, Rozza, Urban. Model Reduction of Parametrized Systems. Springer, 2017.

Breiten and Damm. Krylov subspace methods for model order reduction of bilinear control systems. Systems 86 Control Letters, 59:443-450, 2010.

Carracedo Rodriguez, Gugercin, and Borggaard. Interpolatory model reduction of parameterized bilinear dynamical systems. Submitted to Advances in computational Mathematics, 2017.

Flagg and Gugercin. Multipoint Volterra series interpolation and $\mathcal{H}_{2}$ optimal model reduction of bilinear systems. SIAM Journal on Matrix Analysis and Applications, 36(2):549-579, 2015.

Hesthaven, Rozza, and Stamm. Certified reduced basis methods for parametrized partial differential equations. Springer, 2016.

Quarteroni, Manzoni, Negri. Reduced Basis Methods for Partial Differential Equations. Springer, 2016. 